\title{
VENEZUELA 2013: UN PAÍS A DOS MITADES
}

\author{
Venezuela 2013: A country split in half
}

\section{IÑAKI SAGARZAZU}

Universidad de Glasgow

\section{RESUMEN}

En este artículo se analiza el primer año del chavismo sin Chávez en la política venezolana. El 2013 estuvo marcado no solo por el fallecimiento del presidente reelegido, sino también por lo cerca que estuvo la oposición de obtener la victoria en la elección presidencial, que fue necesario hacer, para elegir al nuevo primer mandatario. Los resultados de este evento electoral marcaron, entonces, la pauta política del año que vio un aumento en la confrontación entre ambos sectores políticos. Después de ver la evolución de la situación del país y los resultados de las elecciones municipales, el artículo concluye analizando cómo las divisiones internas, tanto dentro de la oposición como del chavismo, aunadas al periodo de dos años sin procesos electorales que empieza en 2014, pueden abrir espacios de diálogo o generar mayores enfrentamientos entre ambos sectores.

Palabras clave: chavismo, confrontación, elecciones, oposición, Venezuela.

\begin{abstract}
This article studies the first year of chavismo without Hugo Chávez presence in Venezuelan politics. 2013 was not only marked by the death of the re-elected president Chavez, but also for how close the opposition was to winning the presidential election held to choose a new president. The results of this election set the pattern for the year's political life, which saw an increase in the level of confrontation between the two forces. After taking a look at the countries increasingly troubled economy and safety situation and at the December local elections, the article concludes analyzing the possibilities that open up with the upcoming electoral vacuum.
\end{abstract}

Key words: chavismo, confrontation, elections, opposition, Venezuela. 


\section{INTRODUCCIÓN}

Para no perder la costumbre creada en 14 años desde el año de la victoria electoral de Hugo Chávez en 1998, 2013 ha sido otro año en el que la agenda política en Venezuela dominó nuevamente la escena.

El 2012 cerró con la reelección de Hugo Chávez para un nuevo sexenio, la consolidación del chavismo en el poder regional y la recaída en la salud del primer mandatario (Cyr, 2013). Desde finales del 2012 empezó entonces un periodo de dudas e incertidumbres. El 10 de enero de 2013, día de la toma de posesión presidencial, el acto de juramentación no se pudo realizar debido a la ausencia del reelegido primer mandatario. Una sentencia del 9 de enero emitida por el Tribunal Supremo de Justicia permitió la continuidad del ejecutivo basada en el "principio de continuidad administrativa". De esta forma el gobierno presidido por Hugo Chávez continuaba a pesar de la falta de una toma de posesión para un nuevo mandato. El 5 de marzo el gobierno en voz del vicepresidente ejecutivo, Nicolás Maduro, anunció el fallecimiento de Hugo Chávez. Con esta noticia se cerró un ciclo en la política venezolana moderna. Al mismo tiempo, sin embargo, se activó el mecanismo de transición que la constitución establece, ante la falta absoluta del Presidente en funciones. Así pues, el Consejo Nacional Electoral, en su reunión del 9 de marzo, convocó a elecciones presidenciales anticipadas para el 14 de abril del 2013.

La ausencia de Hugo Chávez de la escena política venezolana no puede tomarse a la ligera. Desde su entrada al poder, la figura de Hugo Chávez fue usada como el elemento cohesionador del movimiento y su carisma fue el elemento principal de su estrategia electoral (Merolla y Zechmeister, 2011; Weyland, 2003). Asimismo, su férreo control y liderazgo permitieron que la coalición chavista se mantuviera cohesionada (Kornblith, 2013), y su imagen polarizadora permitía también la cohesión de la oposición que agrupa diversos elementos unidos en su rechazo al movimiento chavista (Barrionuevo, 2010). Su ausencia por lo tanto representa problemas de coordinación, tanto para el gobierno como para la oposición. Del lado del gobierno, el nombramiento de Nicolás Maduro como heredero directo eliminó cualquier posibilidad de enfrentamiento abierto entre las diferentes tendencias, y le permitió a éste el apoyo de las diferentes corrientes internas para enfrentar las elecciones presidenciales de abril de 2013. Este liderazgo de Maduro, sin embargo, no es permanente, y el apoyo de Chávez no es perfectamente transferible. Del lado opositor, la ausencia de Chávez presenta oportunidades encontradas. Por un lado, la ausencia de un foco polarizador como lo era Hugo Chávez puede ayudar a la debilitación de la unidad opositora; sin embargo, por el otro lado también permite a la oposición elaborar un discurso en el que rechazar la oferta electoral del chavismo deja de ser un rechazo directo a Chávez, intentando romper así el efecto del carisma de Chávez en el comportamiento electoral de sus seguidores.

Para poder entender, entonces, el desarrollo del primer año el chavismo sin Chávez, este artículo empezará con un análisis del primer proceso electoral en 15 años, en el que Hugo Chávez no se presenta como candidato. Posteriormente se evaluará el desgaste que ha sufrido el modelo económico y social del chavismo, y se mostrará cómo este desgaste, todavía, no se termina de observar en el ambiente político. Se concluirá con 
una reflexión sobre el sistema político venezolano, evaluando las nuevas dinámicas que se presentaron en el primer año de ausencia de Hugo Chávez, y cómo esto podría definir la política venezolana a futuro.

\section{LA ELECCIÓN PRESIDENCIAL DE ABRIL DE 2013}

Basándose en el artículo 233 de la Constitución, que establece que ante la falta absoluta del presidente, durante los primeros cuatro años del periodo constitucional, se debe convocar a elecciones anticipadas para elegir un nuevo presidente, el Consejo Nacional Electoral (CNE) procedió, una vez anunciado el fallecimiento del primer mandatario el 5 de marzo, a realizar la convocatoria a elecciones presidenciales. Esta nueva convocatoria tiene la particularidad de ser extremadamente rápida, porque el mismo artículo constitucional establece que las elecciones deben hacerse en los 30 días consecutivos a la declaración de la falta absoluta. Asimismo, debido al vacío legislativo ante este tipo de eventualidades, el CNE toma decisiones para modificar, temporalmente, algunas disposiciones de la Ley de Procesos Electorales, que imposibilitarían el cumplimiento del precepto constitucional. ${ }^{1}$ De esta forma se acortaron todos los procesos relativos al acto electoral, en particular, el período de campaña se redujo a diez días.

Una vez convocadas las elecciones y pasado el período de luto decretado por el gobierno, los actores políticos procedieron a oficializar sus candidaturas. Por el lado del oficialismo se presentó, como dictara el presidente Chávez antes de fallecer, a Nicolás Maduro; del lado opositor, los diferentes miembros de la Mesa de la Unidad decidieron presentar nuevamente a Henrique Capriles. Esta última decisión fue tanto práctica como estratégica. Por un lado, facilitaba el proceso de conocimiento del candidato. Debido a la extensa campaña que realizó Capriles desde las primarias opositoras del 2012, y, pasando por la elección presidencial de octubre de ese año, el gobernador de Miranda era un nombre altamente reconocido en el electorado. Además de esto, el $45 \%$ de votos obtenidos en la elección presidencial de octubre de 2012 (el mejor de cualquier candidato opositor desde 1998) le daba un piso bastante alto de salida. Cambiar de candidato podría significar perder electorado ante la falta de conocimiento del nuevo candidato opositor. Asimismo, nominar a Capriles era estratégicamente positivo para las fuerzas políticas opositoras, que en las primarias estuvieron en su contra (AD, COPEI, UNT, etc.). Esto se debe a que, posterior al fallecimiento del presidente Chávez, era esperado que esto generara un efecto electoral importante en apoyo a su sucesor. Esta inevitabilidad de una victoria de Maduro era avalada por los números que presentaban las distintas encuestadoras. ${ }^{2}$

Durante la campaña, los actores políticos siguieron dos estrategias esperadas. Por un lado, el chavismo utilizó la figura de Chávez para arropar a Maduro, a quien llamó "el hijo de Chávez". En este sentido, la campaña de reforzar la asociación entre Maduro y

Resolución del Consejo Nacional Electoral Nro. 130303-0028 (9 de marzo de 2013).

Por ejemplo, en una encuesta realizada por la firma Datanalisis entre el 11 y el 13 de marzo el partido de gobierno tenía una intención de voto de $49,02 \%$, mientras que la oposición estaba en $34,80 \%$, esto se traduciría en un escenario donde el gobierno obtendría $58,5 \%$ de los votos y la oposición $41,5 \%$. 
Chávez fue un poco problemática, ya que rápidamente fue obvio que Maduro carecía del carisma de su predecesor. Por ejemplo, una serie de comentarios desafortunados hicieron que Nicolás Maduro fuese el centro de burlas dentro de la sociedad venezolana tanto opositora como oficialista. El más claro ejemplo de esto fue el comentario que realizó en el medio de la campaña electoral sobre cómo Chávez se le apareció como un pájaro (El país, 3 de abril de 2013). La estrategia de asociar a Maduro directamente con Chávez estaba presente en todos los aspectos de la campaña electoral del oficialismo. Desde el rediseño de la tarjeta electoral del PSUV para incorporar los famosos ojos de Chávez, pasando por las frases de la campaña como "Chávez te lo juro mi voto es pa'Maduro", que fue usada en el tema de la campaña titulado "Maduro desde mi corazón". 3

Por otro lado, el uso de la figura de Chávez fue utilizado también por la oposición. Su uso estaba dirigido a destacar que Maduro no era Chávez. De esta forma, la oposición aprovechó para atacar de forma intensa a Maduro, sin atacar directamente a Chávez. Este punto es muy importante, porque permitía hablarle al electorado chavista sin atacar a su líder. Para esto, la oposición hizo uso de las múltiples críticas que el mismo Chávez realizó a su equipo de gobierno, y de las decisiones que Maduro tuvo que tomar, como vicepresidente ejecutivo, durante el período en el que el presidente Chávez estaba bajo tratamiento en Cuba. El candidato opositor, Henrique Capriles, cambió su estilo de campaña por uno más confrontacional. En los mítines de campaña, organizados en las diferentes ciudades, era común escuchar a Capriles diciendo frases como "El problema eres tú, Nicolás" (BBC Mundo. 22 de marzo de 2013).

Los efectos de estas dos campañas fueron muy claros, y la secuela positiva que podría haber tenido el fallecimiento de Hugo Chávez, en la intención de voto de Maduro, rápidamente desapareció. La Figura 1 da cuenta de la drástica evolución de la intención de voto; como se puede observar en un lapso de un mes esta pasa desde un promedio de $60 \%$ hasta cerca del $50 \%$.

A pesar del efecto claro de la campaña, es importante resaltar también que la situación del país venía deteriorándose después de la elección presidencial del 2012. Según la encuestadora Datanalisis, entre diciembre de 2012 y marzo de 2013 la evaluación negativa del gobierno pasó de 38,3\% a 56,6\%. ${ }^{4}$ Esto se debe en gran parte a un aumento en los problemas que aquejan a los ciudadanos. Por ejemplo, en febrero de 2013 el 49,7\% de los entrevistados manifestó que su principal problema era la inseguridad. ${ }^{5}$ La creciente percepción de que los problemas aumentaban, junto con el aumento en la evaluación negativa de la gestión gubernamental, ayudaron a que la estrategia de confrontación de Henrique Capriles rindiera frutos pasando de una brecha de 20 puntos a una de 5 puntos.

Un componente adicional de la campaña electoral fue el desbalance del árbitro electoral. Esto se evidenció en una rapidez para sancionar la campaña opositora, mientras, al mismo tiempo, se permitían abusos por parte del gobierno. Por ejemplo, la campaña

\footnotetext{
http://www.psuv.org.ve/audios/maduro-desde-mi-corazon/

Encuesta Nacional Omnibus, julio 2013.

Encuesta Nacional Omnibus, julio 2013.
} 
de Maduro utilizó recursos gubernamentales, desde actos de campaña transmitidos en cadena nacional hasta el uso de instalaciones del gobierno para promover la campaña de Maduro. Esta práctica, que ya existía del pasado, fue incrementada para asegurar la continuidad de la revolución. ${ }^{6}$ Este uso indebido de los recursos del Estado fue denunciado por la ONG Transparencia Venezuela ante el CNE, el 9 de abril de 2014 (El Universal, 1 de abril de 2013), y fue altamente ignorado por los rectores del CNE, según denunció uno de los miembros de este cuerpo colegiado (El Universal, 1 abril 2013).

Figura 1. Evolución de la intención de voto - Elecciones presidenciales abril 2013

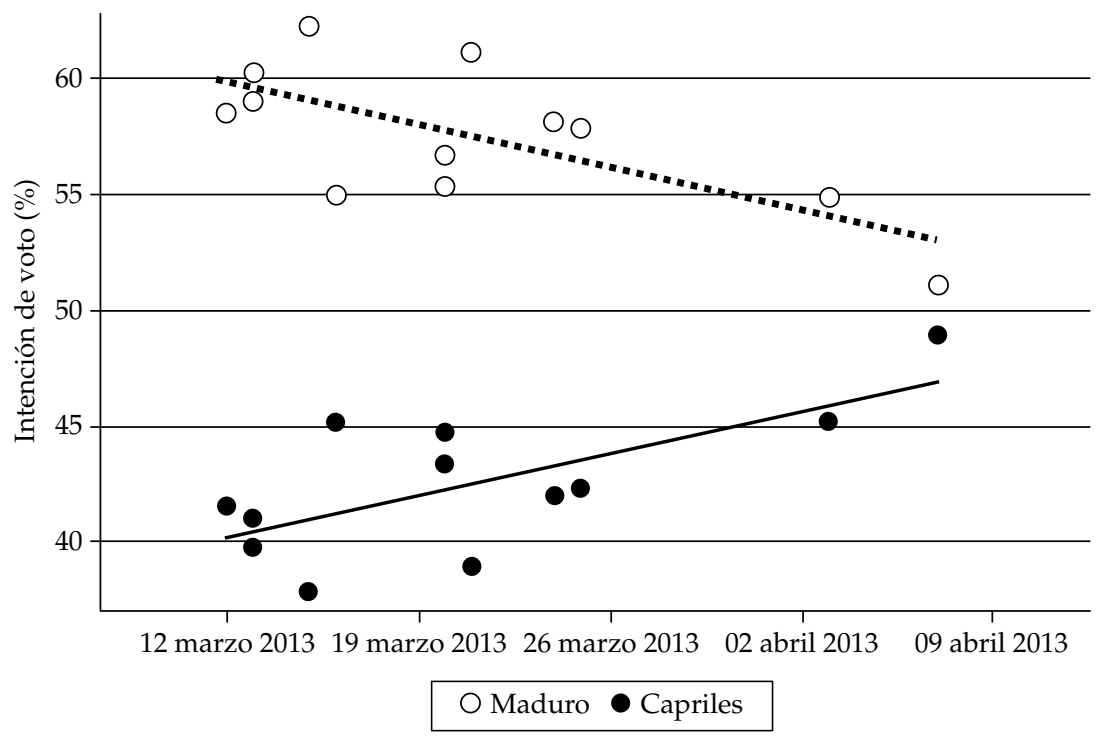

Fuente: Elaboración propia.

Después de un período muy intenso de campaña electoral, los venezolanos volvían a tornarse a las urnas para elegir un Presidente, a menos de un año de la elección presidencial anterior. Una vez más, los resultados de las urnas favorecían al partido de gobierno. Sin embargo, en esta oportunidad, la diferencia era de menos de dos puntos porcentuales (la Tabla 1 muestra los resultados electorales). A pesar de obtener una mínima mayoría, Nicolás Maduro, había logrado perder un porcentaje importante de votos, ya que con una tasa de participación similar, Maduro obtuvo cinco puntos porcentuales menos que Hugo Chávez.

6 El reporte de la Misión de Observación del Centro Carter en las elecciones presidenciales de 2012 ya recogía estas críticas (Carter Center, octubre 2012). 
Tabla 1. Resultados electorales de elecciones presidenciales 2012 y 2013

\begin{tabular}{lcc}
\hline & Octubre 2012 & Abril 2013 \\
\hline \multirow{2}{*}{ Chavismo } & Hugo Chávez & Nicolás Maduro \\
& $55,07 \%$ & $50,61 \%$ \\
Oposición & Henrique Capriles & Henrique Capriles \\
Participación & $44,31 \%$ & $49,12 \%$ \\
& $15,160,289$ & $15,057,480$ \\
& $80,49 \%$ & $79,68 \%$ \\
\hline
\end{tabular}

Fuente: Consejo Nacional Electoral (CNE).

Como se puede observar en la Tabla 1, los resultados electorales fueron considerablemente cercanos. Igual que sucedió en las presidenciales de 2012, la participación fue muy alta, llegando al 80\% del padrón electoral. Esto se traduce en que, en la gran mayoría de centros de votación, no hubo mucha diferencia entre la participación de una y otra elección. Sin embargo, lo mismo no puede decirse sobre el apoyo a Maduro y Capriles. Si comparamos la elección de 2013 con la presidencial de 2012, podemos observar que el cambio de 5 puntos porcentuales observado a nivel nacional viene dado por un cambio similar a nivel local. La Figura 2 muestra la distribución de la diferencia entre el porcentaje de votos obtenido por Maduro comparado con Chávez (en 2012), la diferencia entre los votos obtenidos por Capriles y la diferencia en el porcentaje de participación, todo a nivel de mesa electoral. Como se puede observar, en general el promedio en diferencia de votos entre Maduro y Chávez es 5\%, y como era de esperar, un aumento del 5\% para Capriles. Sin embargo, la Figura 2 muestra, también, que hubo un número alto de centros electorales donde la diferencia entre una elección y otra fue mayor.

Después de una larga espera por los resultados electorales, el CNE en voz de su presidenta Tibisay Lucena, anuncia la victoria de Nicolás Maduro. Ante este anuncio el candidato de la opositora Mesa de la Unidad, Henrique Capriles, anunció, en rueda de prensa, que no reconocería los resultados hasta tanto no se realizara un conteo voto por voto (El País, 15 de abril 2013). Con este anuncio, Venezuela volvía a la incertidumbre que existió posterior al Referéndum Revocatorio de 2004. Las acciones posteriores, tanto de parte del gobierno como de la oposición, fueron bastante rápidas. De parte del CNE, se procedió a la inmediata certificación de los resultados mediante la proclamación de Nicolás Maduro como Presidente electo, el día 15 de abril (un día después de las elecciones). La oposición, por su parte, entregó, dos semanas después de realizado el acto de votación, la impugnación del proceso ante el Tribunal Supremo de Justicia (TSJ). ${ }^{7}$

7 En decisiones del 7 de agosto de 2013 el TSJ rechazó las diferentes impugnaciones al proceso electoral introducidas tanto por la Mesa de la Unidad y su candidato Henrique Capriles Radonski como por otros actores. 
Figura 2. Comparación de resultados a nivel de mesa electoral

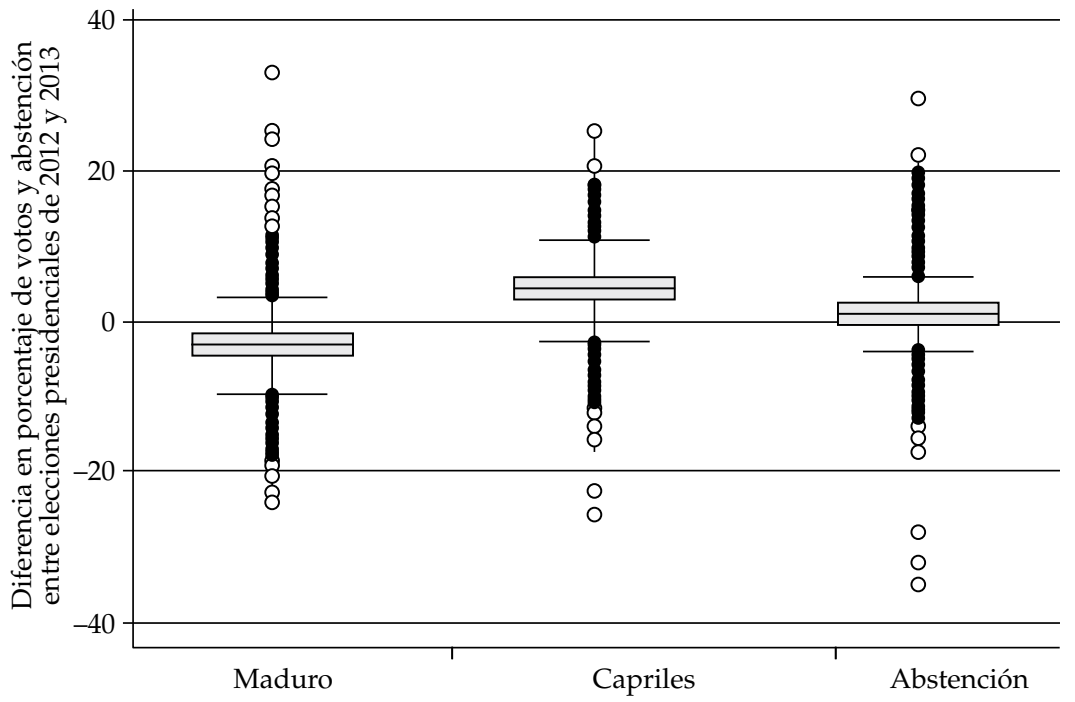

Fuente: esdata.info

El rechazo de la oposición de reconocer, inicialmente, a Nicolás Maduro como Presidente, generó múltiples tensiones entre las dos fuerzas políticas. En los días posteriores al proceso electoral el gobierno acusó a la oposición de organizar los focos de violencia que surgieron, en diversas ciudades del país, en protesta por los resultados, en particular, responsabilizando a Henrique Capriles de estos hechos. Asimismo, el gobierno presionó a los gobernadores de oposición a reconocerlo como presidente, ya que, de lo contrario, él dejaría de reconocerlos como gobernadores (RCN Noticias, 16 abril 2013). Igualmente desde el Parlamento, el presidente de la Asamblea Nacional, Diosdado Cabello, comunicó que los diputados que no reconocieran a Nicolás Maduro no tendrían derecho de palabra en el hemiciclo, y destituyó a los presidentes de comisiones opositores negados a reconocer al nuevo ejecutivo (El Espectador, 7 mayo 2013).

\section{UN MODELO ECONÓMICO Y SOCIAL QUE MUESTRA SEÑALES DE DESGASTE}

El 2013 no solo empezó con un gobierno cuyo Presidente reelegido estaba muy enfermo como para poder asistir a su investidura, sino también con una situación económica necesaria de ser atendida. La inyección de recursos en la economía, como parte de la campaña electoral presidencial de 2012, comenzó a mostrar sus efectos a finales del 2012, con unos índices de inflación mensual de más de $2 \%$ en los últimos tres meses (según las cifras oficiales del Banco Central de Venezuela - BCV). Estos problemas económicos se vieron reflejados también en la devaluación del bolívar, que el gobierno aprobó en febrero de 2013 (pasando de 4,30 a 6,30 bolívares por dólar). La devaluación, a su vez, 
encareció los precios, y en abril de 2013 ya la inflación mensual alcanzaba 6\% (ver Figura 3). Para controlar este fenómeno, el gobierno intentó implementar un mayor control de precios, e inclusive, llegó a fijar niveles de ganancia por la Ley Habilitante, aprobada por la Asamblea Nacional (Minci, 19 noviembre 2013). Con esta ley, el gobierno pudo investigar e intervenir empresas que eran identificadas como incursas en usura y especulación (El Universal, 8 noviembre 2013; Panorama, 14 noviembre 2013).

Los problemas económicos, sin embargo, no eran los únicos que afectaban a los venezolanos. Ya en marzo del 2013 Venezuela estaba entre los diez países del mundo con mayor número de secuestros y homicidios (El Universal, 17 marzo 2013). Para combatir esta problemática, el gobierno activó, a mediados del año, el Plan Patria Segura, en el marco de la Misión A Toda Vida Venezuela, iniciada en 2012 por el expresidente Chávez. Con este plan, los miembros de las Fuerzas Armadas se sumarían a los organismos de seguridad, ya en la calle (Telesur, 13 mayo 2013; MINCI, 17 mayo 2013). Según cifras oficiales, a pesar de haber una reducción en la tasa de homicidios, comparado con el primer trimestre del año, la criminalidad seguía alta (El Universal, 28 diciembre 2013).

Además de los problemas económicos, evidenciados en el aumento de la inflación, y de la problemática social, que se presenta con el aumento de los índices de delincuencia y la sensación de inseguridad, el gobierno de Maduro tuvo que lidiar desde principios de año con serios problemas de abastecimiento de productos de primera necesidad. El más claro ejemplo de esta problemática se dio en mayo del 2013 cuando el gobierno se vio forzado a realizar una muy pública importación de papel higiénico debido a la ausencia de este producto en el mercado (Time, 17 mayo 2013).

Figura 3. Índice Nacional de Precios al Consumidor (2010-2013)

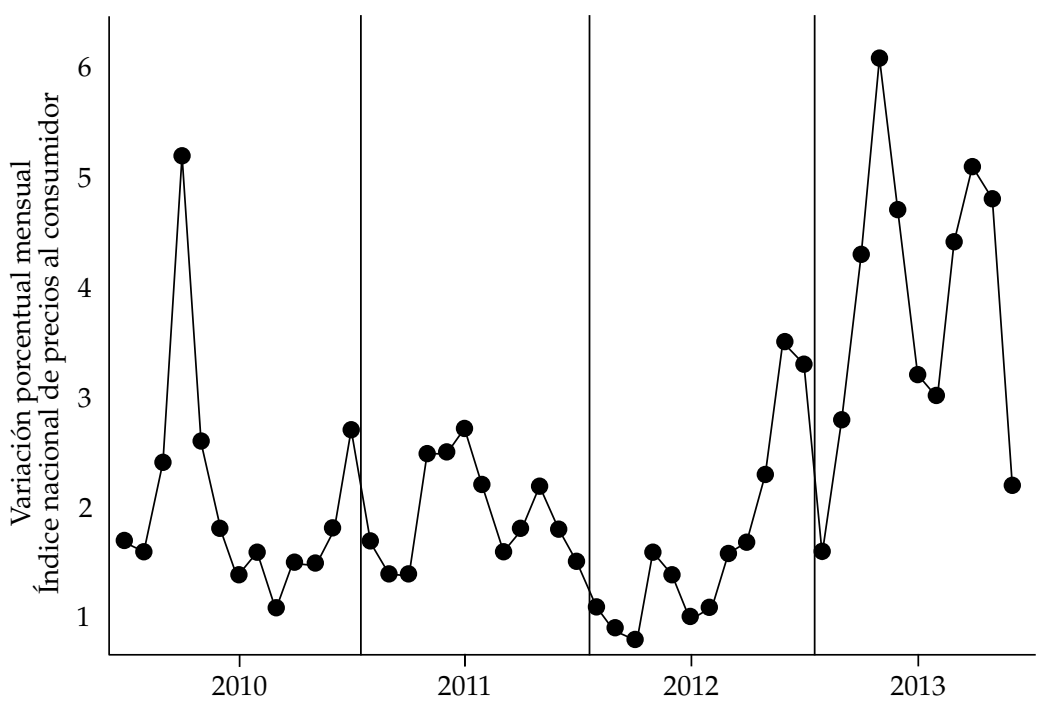

Fuente: Banco Central de Venezuela. 
Como es de esperar, el aumento de los problemas afectó, directamente, los niveles de aprobación de la gestión del gobierno. En la Figura 4 se puede observar la evolución de la evaluación positiva de gestión del gobierno y la tendencia promedio de estas. ${ }^{8}$ Sin embargo, es importante destacar que esta disminución es bastante leve, ya que llega solamente a 5 puntos en promedio en más de seis meses. Considerando el gran aumento de la inflación durante el año y los altos niveles de inseguridad, entre otros problemas, que la disminución haya sido tan leve es bastante impresionante. Esto tiene sentido si consideramos los factores que entran en la evaluación de gestión del chavismo. Un estudio con los datos del Proyecto de Opinión Pública en América Latina (LAPOP) ${ }^{9}$ muestra cómo la evaluación presidencial depende, primordialmente, de la evaluación económica y de la participación en las misiones sociales. Esta última es responsable de un aumento de 20 puntos porcentuales en la evaluación de gestión (Pérez, 2013). Basado en estos hallazgos del comportamiento político del venezolano en los últimos años, podemos explicar la disminución de la popularidad a base del incremento de los problemas que afectan al venezolano; $y$ a la vez, podemos entender cómo esta reducción es tan leve, a base de la alta participación de los venezolanos en las misiones sociales.

Figura 4. Evolución de la evaluación positiva de gestión

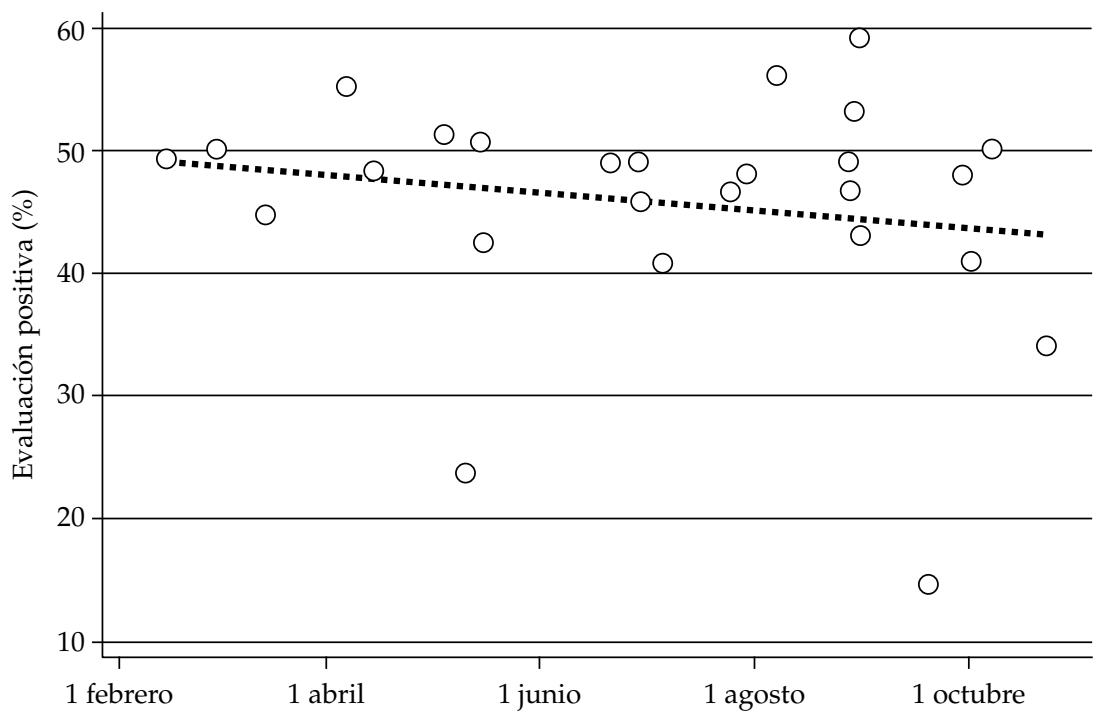

Fuente: Elaboración propia.

8 Las encuestas presentadas son de diferentes casas encuestadoras. La alta variación entre los porcentajes está relacionada con las diferentes encuestadoras y los sesgos que cada casa encuestadora tiene, más que con una falta de consistencia del electorado. Un análisis de las diferencias entre las diferentes encuestadoras muestra cómo el sesgo progobierno o prooposición de las encuestadoras afecta los valores de aprobación de gestión (Sagarzazu, 2013).

9 Latin American Public Opinion Project (LAPOP). 


\section{ELECCIONES MUNICIPALES DE DICIEMBRE DE 2013}

El segundo proceso electoral del año, y originalmente el único que correspondía, eran las elecciones municipales de alcaldes y legislativos locales (concejos municipales). Estas elecciones, originalmente, deberían haberse realizado junto a las elecciones de gobernadores de 2012; sin embargo, el CNE decidió en el 2012 separar los tres procesos electorales (presidenciales, regionales y municipales). En total en este proceso se eligieron 337 alcaldes y 2.457 concejales.

Para este proceso electoral ni la oposición ni el chavismo fueron completamente unificados. De parte de la oposición, a pesar de haber realizado las primarias en 2012 para seleccionar candidatos unitarios, hubo lugares en los que se rompió la unidad. Sin embargo, fueron partidos pequeños los que hicieron esto. Entre los partidos principales solo el Movimiento al Socialismo (MAS) presentó candidatos fuera de los que surgieron de las primarias, ningún otro de los principales partidos de la oposición rompió filas; esto, a pesar de que hubo varios casos donde se intentó cambiar los candidatos respecto de los elegidos en las primarias. ${ }^{10}$ Del lado oficialista, las candidaturas fueron decididas por la cúpula del partido obviando a las bases. En algunos casos, como la alcaldía de los municipios Baruta y Sucre del estado Miranda o la alcaldía de Maracaibo en el estado Zulia, los candidatos no provenían ni de las filas del partido de gobierno ni de la comunidad, sino más bien del mundo del espectáculo.

Durante la campaña, la oposición utilizó nuevamente como estrategia la confrontación directa con Maduro. En particular, se intentó utilizar la creciente insatisfacción con el gobierno, para usar las elecciones como un plebiscito contra Maduro. Para la oposición, que sigue argumentando que los resultados de la elección presidencial del 14 de abril no eran correctos, era importante ganar numéricamente a nivel nacional. Para ello utilizaron una estrategia similar a la de las presidenciales y nombraron a Henrique Capriles jefe nacional de la campaña de la oposición (El País, 4 diciembre 2013).

Al finalizar la jornada electoral se evidenció, una vez más, el predominio del chavismo. En unos resultados -globales- que supusieron buenas noticias para el primer mandatario, el oficialismo obtuvo cerca del $54 \% .{ }^{11}$ Este porcentaje de votos lleva al chavismo de regreso al promedio que habían visto en las dos elecciones del 2012 (ver Tabla 2). Por su parte, la oposición retrocede de los excelentes resultados que obtuvo en las elecciones presidenciales del 2013. Sin embargo, en número de alcaldías aumenta de las 56 que tenía posterior a las elecciones de 2008 a 77. Tal vez, más importante aún, es que la oposición logró victorias importantes en las principales ciudades del país, manteniendo todas las

10 Una excepción es el municipio El Hatillo del estado Miranda donde la alianza unitaria permitió a los diferentes partidos presentarse individualmente. Esta flexibilización se debió a dos factores: 1) el candidato seleccionado en las primarias falleció poco tiempo después de celebradas estas; 2) la votación opositora en este municipio es típicamente cercana al $90 \%$ y por lo tanto la oposición no consideró que existía la posibilidad de perder contra el candidato del gobierno. Por ejemplo en las elecciones presidenciales Capriles obtuvo un poco más de 32.000 votos y Maduro 7.000.

11 Entre votos a la tarjeta electoral del PSUV como a las de los partidos que conforman la alianza oficialista. 
alcaldías que ya tenía en el área metropolitana de Caracas $^{12}$ y la alcaldía de Maracaibo en el estado Zulia. A esto agregaron alcaldías importantes, como la alcaldía de Valencia (estado Carabobo), la alcaldía de Iribarren (estado Lara) de la ciudad de Barquisimeto, y la alcaldía de San Cristóbal (estado de Táchira).

El hecho de que la oposición gane en ciudades importantes no es sorpresa, ya que las clases medias y altas son el principal sustento opositor y estas se ubican en las zonas urbanas. Lo que es más importante es que la oposición gana terrenos importantes desde donde tener cuotas de poder que hasta ahora le rehuían o habían perdido. Lo que demuestran los resultados también es que el chavismo sigue teniendo un férreo control sobre las zonas rurales y que el mapa político, en el que la oposición tiene problemas para nacionalizarse eficientemente, todavía existe (Sagarzazu, 2011). Así pues vemos cómo la oposición obtiene victorias importantes en los estados andinos, donde COPEI (Táchira) y AD (Mérida y Trujillo) siempre fueron importantes; en el Zulia (UNT), en Miranda (Primero Justicia), y en Carabobo (Proyecto Venezuela). A esto se suma el estado Lara y el estado Amazonas donde gobiernan exchavistas que provenían originalmente de las filas del PPT y otras municipalidades importantes en estados como Maturín, Barinas que sí serían verdaderas incursiones en terreno chavista.

\section{CONCLUSIÓN}

Como se puede observar, el 2013 fue nuevamente un año bastante activo en el frente político en Venezuela. El fallecimiento de Hugo Chávez, a principios de año, abrió un clima de incertidumbre sobre la continuación del proceso Bolivariano. Esta incertidumbre incrementó con el resultado de la elección presidencial de abril, en la que la oposición, a pesar de jugar en un terreno altamente desfavorable, tanto en términos de recursos como en términos institucionales, ${ }^{13}$ aunado al factor emocional del fallecimiento de Chávez, logró estar a menos de 2 puntos porcentuales de obtener la victoria. ${ }^{14}$

Tabla 2. Resultados electorales 2012-2013

\begin{tabular}{lcccc}
\hline & $\begin{array}{c}\text { Presidenciales } \\
\text { octubre 2012 }\end{array}$ & $\begin{array}{c}\text { Regionales } \\
\text { diciembre 2012 }\end{array}$ & $\begin{array}{c}\text { Presidenciales } \\
\text { abril 2013 }\end{array}$ & $\begin{array}{c}\text { Municipales } \\
\text { diciembre 2013 }\end{array}$ \\
\hline Chavismo & $55,07 \%$ & $54,5 \%$ & $50,61 \%$ & $53,87 \%$ \\
Oposición & $44,31 \%$ & $44,78 \%$ & $49,12 \%$ & $43,26 \%$ \\
Participación & $80,49 \%$ & $53,94 \%$ & $79,68 \%$ & $58,92 \%$ \\
\hline
\end{tabular}

Fuente: ${ }^{1}$ Consejo Nacional Electoral (CNE) ${ }^{2}$ esdata.info ${ }^{3} \mathrm{El}$ Universal (15 diciembre 2013).

12 Que incluye las alcaldías de Sucre, Baruta, El Hatillo y Chacao (estado Miranda) y la Alcaldía Mayor de Caracas. La única alcaldía de Caracas donde el oficialismo todavía es mayoría es la del municipio Libertador (Distrito Capital).

13 Corrales y Penfold (2007) presentan un análisis sobre cómo estos factores se han presentado e incrementado desde 1999.

14 A pesar de que los múltiples recursos introducidos han sido rechazados, Henrique Capriles todavía mantiene la posición de que la elección fue fraudulenta. 
Este resultado tan cercano tuvo también efectos dentro del chavismo. Pasar de $60 \%$ de apoyo a $50 \%$ en un mes, por los errores cometidos en la campaña, generó la sensación de que Maduro había malgastado el legado de Chávez, dificultando así el control que Maduro podría ejercer sobre las diferentes facciones del chavismo (Corrales, 2013). Este problema de legitimidad interna dificultó los primeros meses del gobierno de Maduro, en particular en términos de intentar solucionar la creciente problemática social. Por el contrario, en un intento por recuperar, en las elecciones municipales, el caudal de votos perdidos en abril, el chavismo navegó, durante el resto del 2013, en una repetición de las políticas públicas de intervención en la economía que ya le habían rendido frutos en el pasado. Después de la victoria en las municipales, por casi 10 puntos porcentuales a nivel nacional, el gobierno volvió, entonces, al promedio de apoyo que había acumulado en el 2012, y Maduro se vio reforzado. Lo más importante, sin embargo, es que con las elecciones municipales se culminó un ciclo bastante cargado electoralmente (con cuatro elecciones en dos años), y se empezó un nuevo período, atípico, sin elecciones por dos años. ${ }^{15}$

Este período sin elecciones genera dilemas, tanto para el gobierno como para la oposición, pero también presenta oportunidades para ambos. Por el lado de la oposición es difícil mantener la motivación de sus seguidores sin procesos electorales que los esperancen. Asimismo, el retroceso electoral que representaron las elecciones municipales tiene la posibilidad de reabrir viejas grietas dentro de la poco cohesionada unidad opositora. Es importante recordar que la oposición es una mezcla de diversos actores políticos desde partidos de izquierda como Bandera Roja hasta movimientos de derecha. Además de la diversidad ideológica la oposición también está dividida en cómo plantarse ante el gobierno, con actores más moderados que buscan vías de diálogo y una canalización electoral del conflicto (como el candidato Henrique Capriles) y otros más radicales que consideran opciones más allá de la electoral como por ejemplo la protesta activa en las calles (como la diputada María Corina Machado y el exalcalde Leopoldo López). Todos estos factores están unidos únicamente por abogar por la finalización del periodo de gobierno chavista. Esta división en términos de estrategia es la que más problemas ha presentado al liderazgo opositor y la que ha dictado su comportamiento. Así pues la historia de la oposición ha sido un constante cambio en el dominio entre estas dos fuerzas. Desde el fracaso de la estrategia radical en los años 2002-2005 el grupo de moderados tomó las riendas del movimiento e intentaron vencer al oficialismo en el plano electoral. Ante la inhabilidad del sector moderado (que viene liderando desde 2006) de lograr una victoria electoral es altamente posible que los sectores más radicales intenten retomar el liderazgo opositor.

Por otro lado, el vacío electoral permite al liderazgo opositor entablar una conversación con el gobierno para tratar de solventar conjuntamente algunos de los problemas que aquejan a los venezolanos. Esto último, sin embargo, requiere que el gobierno abra vías reales de diálogo con los sectores opositores. El reconocimiento por parte del gobierno de las victorias de la oposición y la convocatoria a los alcaldes y gobernadores opositores al 
Palacio Presidencial de Miraflores apuntan ciertamente en esa dirección (Panorama, 19 diciembre 2013). Precisamente es el gobierno quien sale más beneficiado por el espacio que se abre sin elecciones, porque le permite abrirse al diálogo e intentar aplicar medidas necesarias para solventar la problemática económica y social que se ha venido acumulando en los últimos años, soluciones que, por su impopularidad, no han sido implementadas.

A pesar de los espacios que se abren para el diálogo y la resolución de los problemas que aquejan a los venezolanos, esto solo será posible si los moderados, dentro de la oposición y los de dentro del gobierno, logran imponerse. De cierta forma, y a pesar de que Venezuela actualmente tiene un régimen democrático categorizado como "autoritarianismo competitivo" (Levitsky y Way, 2010; Cyr, 2012), la dinámica política venezolana actual es similar al concepto de coup poker que O'Donnell y Schmitter (1986) introducen para explicar transiciones de regímenes autoritarios a democráticos. En este juego, los moderados y radicales de ambos bandos tienen diferentes incentivos, fuerzas y metas; la interacción entre estos cuatro actores puede recrudecer el régimen autoritario, generar una transición, o generar un cambio de un régimen autoritario por otro (Crescenzi, 1999). En este sentido, si los radicales de uno de los dos bandos ven el espacio que se abre sin elecciones y se imponen sobre los moderados de su bando, esto podría tener una reacción similar del lado opuesto, lo que llevaría a Venezuela a una situación parecida a la de los años 2002-2004.

\section{REFERENCIAS}

Barrionuevo, Jorge. 2010. "Elecciones primarias en Venezuela. La MUD y los líos con el CNE". Revista Andina de Estudios Políticos 5: 3-13.

BBC Mundo. 22 de marzo de 2013. Venezuela: Capriles calienta motores contra «Nicolás». http:/ /www. bbc.co.uk/mundo/noticias/2013/03/130321_venezuela_elecciones_2013_oposicion_henrique_ capriles_inicio_precampana_az.shtml [consulta 25 febrero 2013]

Carter Center. October, 2012. Study Mission to the October 7, 2012 Presidential Election in Venezuela. http://www.cartercenter.org/resources/pdfs/news/peace_publications/election_reports/venezuela2012-election-study-mission-final-rpt.pdf [ consulta 25 febrero 2013]

Corrales Javier y Michael Penfold. 2007. "Venezuela: Crowding Out the Opposition”. Journal of Democracy 18 (2): 99-113.

Corrales, Javier. 2013. “¿Un Maduro Más Duro? Venezuela After Chávez”. Berkeley Review of Latin American Studies. Spring: 16-24.

Crescenzi, Mark J. C.: 1999. "Violence and Uncertainty in Transitions". The Journal of Conflict Resolution 43 (2): 192-212.

Cyr, Jennifer. 2013. “Que veinte años no es nada: Hugo Chávez, las elecciones de 2012 y el continuismo político venezolano". Revista ciencia política 33 (1): 375-391.

El Espectador. 7 mayo 2013. Oposición venezolana podrá hablar solo si reconoce a Maduro como presidente http://www.elespectador.com/noticias/elmundo/articulo-420739-oposicion-venezolana-podrahablar-solo-si-reconoce-maduro-presid [Consultado 26 febrero 2014]

El País. 2 abril 2013. "Maduro dice que un "pajarito" con el espíritu de Chávez lo bendijo" http:/ / internacional.elpais.com/internacional/2013/04/02/actualidad/1364930548_441291.html [Consulta 24 febrero 2014]

El País. 15 de abril de 2013. "Maduro se atribuye la victoria y Capriles rechaza los resultados" http:// internacional.elpais.com/internacional/2013/04/15/actualidad/1366004151_006193.html [Consulta 25 febrero 2014] 
El País. 4 diciembre 2013. Capriles afronta las municipales como un plebiscito contra Maduro. http:/ / internacional.elpais.com/internacional/2013/12/04/actualidad/1386186413_709721.html [Consulta 26 febrero 2014].

El Universal. 17 marzo 2013. Venezuela es el octavo país con mayor número de secuestros. http:/ /www. eluniversal.com/sucesos/130317/venezuela-es-el-octavo-pais-con-mayor-numero-de-secuestros [Consulta 27 febrero 2014].

El Universal. 1 de abril de 2013. "Si CNE no para los abusos sería dramático para la democracia" http:/ / www.eluniversal.com/nacional-y-politica/130401/si-cne-no-para-los-abusos-seria-dramatico-parala-democracia [Consulta 25 de febrero 2014].

El Universal. 8 de noviembre 2013. Detenidos en el Sebin gerentes de Tiendas Daka http:/ / www.eluniversal. com/economia/131108/detenidos-en-el-sebin-gerentes-de-tiendas-daka [Consulta 27 febrero 2014]

El Universal. 15 de diciembre 2013. Chavismo ganó el voto nacional el 8D http:/ / www.eluniversal.com/ nacional-y-politica/elecciones-2013/131215/chavismo-gano-el-voto-nacional-el-8d [Consulta 24 febrero 2014].

El Universal. 28 diciembre 2013. Rodríguez Torres: Tasa de homicidios es de 39 por cada 100 mil habitantes. http:/ / www.eluniversal.com/sucesos/131228/rodriguez-torres-tasa-de-homicidios-es-de-39-porcada-100-mil-habitant [Consulta 28 febrero 2014].

Merolla, Jennifer L. y Elizabeth Zechmeister. 2011. “The Nature, Determinants, and Consequences of Chávez's Charisma: Evidence from a Study of Venezuelan Public Opinion". Comparative Political Studies 44 (1): 28-54.

MINCI - Ministerio del Poder Popular para la Comunicación e Información. 17 mayo 2013. Plan Patria Segura permitirá estructurar un sistema permanente de protección y patrullaje. http:/ /www.sibci. gob.ve/2013/05/plan-patria-segura-permitira-estructurar-un-sistema-permanente-de-protecciony-patrullaje/ [Consulta 28 febrero 2014].

MINCI - Ministerio del Poder Popular para la Comunicación e Información. 19 de Noviembre 2013. Ley Habilitante permitirá regulación de niveles de ganancia. http:/ / www.minci.gob.ve/2013/11/leyhabilitante-permitira-regulacion-de-niveles-de-ganancia/ [Consulta 27 febrero 2014].

O'Donnell, Guillermo y Philippe Schmitter. 1986. Transitions from authoritarian rule: Tentative conclusions about uncertain democracies. Baltimore: John Hopkins University Press.

Panorama. 14 noviembre de 2013. "Máxima ganancia será entre 15\%, como mínimo, y máximo 30\%": Maduro. http:/ / panorama.com.ve/portal/app/push/noticia88738.php [Consulta 27 febrero 2014].

Panorama. 19 diciembre 2013. Maduro a líderes opositores: “Ojalá algún día podamos encontrar puntos de coincidencia" http://panorama.com.ve/portal/app/push/noticia92469.php [Consulta 27 febrero 2014].

Pérez, Orlando J. 2013. "The Basis of Support for Hugo Chávez: Measuring the Determinants of Presidential Job Approval in Venezuela". The Latin Americanist 57: 59-84.

RCN Noticias. 16 abril 2013. Maduro amenaza a gobernadores que no lo reconozcan. http://www. canalrcnmsn.com/noticias/maduro_amenaza_gobernadores_que_no_lo_reconozcan?page=12 [Consulta 26 febrero 2014].

Sagarzazu, Iñaki. 2011. "Nación vs. región: las tensiones del sistema de partidos venezolano postcolapso". América Latina Hoy 58: 121-142.

Sagarzazu, Iñaki. 2014. El problema de la MUD?. YVpolis, [blog] 14 nov 2013 http://yvpolis.blogspot. co.uk/2013/11/el-problema-de-la-mud.html [Consulta 28 febrero 2014].

Telesur. 13 mayo 2013. Presidente de Venezuela activa Plan Patria Segura. http://www.telesurtv.net/ articulos/2013/05/13/presidente-de-venezuela-activa-plan-patria-segura-3840.html [Consulta 28 febrero 2014].

Time. 17 mayo 2013. Bathroom Blues: Venezuela's Toilet-Paper Crisis http:/ /newsfeed.time.com/2013/05/17/ bathroom-blues-venezuelas-toilet-paper-crisis/ [Consulta 21 marzo 2014].

Weyland, Kurt. 2003. "Economic voting reconsidered. Crisis and charisma in the election of Hugo Chavez". Comparative Political Studies 36 (7): 822-848.

Iñaki Sagarzazu es profesor de Política Comparada en la Universidad de Glasgow (Reino Unido). E-mail: Inaki.Sagarzazu@glasgow.ac.uk 